\title{
Relación entre la capacidad de agencia de autocuidado y la calidad de vida en personas con insuficiencia cardiaca*
}

\author{
Relationship Capacity of Self-Care Agency and Quality of Life in People with Heart Failure \\ Relação entre capacidade de agência do autocuidado e qualidade de vida em pessoas com insuAciência cardíaca
}

Diana Yiseth Molano Barrera ${ }^{\text {a }}$

Clinica Universitaria Colombia, Colombia

DOI: https://doi.org/10.11144/Javeriana.ie22.rcaa

dymolanob@unal.edu.co

ORCID: https://orcid.org/0000-0001-7579-1159

Recibido: 25 Enero 2020

Renata Virginia González Consuegra

Universidad Nacional de Colombia, Colombia

ORCID: https://orcid.org/0000-0001-9269-7789

Aceptado: 10 Junio 2020

Publicado: 29 Diciembre 2020

\section{Resumen:}

Introducción. La persona con insuficiencia cardiaca enfrenta cambios biopsicosociales que deterioran su calidad de vida. Es necesario conocer la relación existente entre el autocuidado y la calidad de vida, lo que puede orientar al profesional de enfermería en el diseño de intervenciones efectivas. Objetivo. Determinar la relación existente entre la capacidad de agencia de autocuidado y la calidad de vida relacionada con la salud en las personas con insuficiencia cardiaca, que son atendidas en el programa multidisciplinario de insuficiencia cardiaca en una institución de salud de cuarto nivel en Bogotá, Colombia. Método. Estudio descriptivo correlacional de corte transversal, realizado entre mayo y agosto de 2018; utilizando los instrumentos Appraisal of Self-care Agency Scale y el Cuestionario de Cardiomiopatía de Kansas City; la muestra correspondió a 107 pacientes, mayores de edad, con insuficiencia cardiaca estadio C y D. Resultados. La capacidad de agencia de autocuidado se encontró en categorías alta con $63,55 \%$ y muy alta con $34,57 \%$; la calidad de vida relacionada con la salud se encontró preservada, con un puntaje general de 73,33; la relación existente entre las dos variables, según el coeficiente de correlación de Spearman, fue 0,316 con un valor $\mathrm{p}=0,002$, relación débil pero significativa. Conclusiones. Existe una relación entre las variables de interés, que se reafirma con las correlaciones significativas identificadas entre las dimensiones que las conforman. Estos hallazgos resaltan la pertinencia de abordar, en las intervenciones, temáticas que fortalezcan la capacidad de agencia de autocuidado, contribuyendo a mejorar la calidad de vida de estas personas. Palabras clave: autocuidado, calidad de vida, insuficiencia cardiaca.

\section{Abstract:}

Introduction. The person with heart failure faces biopsychosocial changes that impair their quality of life. It is necessary to know the relationship between self-care and quality of life, which can guide the nursing professional in the design of effective interventions. Objective. To determine the relationship between the capacity of self-care agency and healthrelated quality of life in people with heart failure who are treated in the multidisciplinary program of heart failure in a fourth level health institution in Bogotá, Colombia. Method. Descriptive study correlational, cross-sectional, made between May and August 2018; using the Appraisal of Self-care agency Scale Instruments and the Kansas City Cardiomyopathy Questionnaire; the sample corresponded to 107 patients, of legal age, with stage C and D heart failure. Results. The capacity of self-care agency was in high category with $63.55 \%$ and very high with $34.57 \%$; the health-related quality of life was preserved, with an overall score of 73.33; the relationship between the two variables, according to the Spearman correlation coefficient, was 0.316 with a value $\mathrm{p}=0.002$, a weak but significant relationship. Conclusions. There is a relationship between the variables of interest, which is reaffirmed with the significant correlations identified between the dimensions that comprise them. These findings highlight the relevance of addressing, in the interventions, issues that strengthen the capacity of self-care agency, contributing to improve the quality of life of these people. Keywords: self-care, quality of life, heart failure.

\section{Resumo:}

Introdução. A pessoa com insuficiência cardíaca enfrenta mudanças biopsicossociais deteriorando sua qualidade de vida. É preciso conhecer a relação existente entre autocuidado e qualidade de vida, o que pode nortear o profissional de enfermagem

Notas de autor

a Autora de correspondencia. Correo electrónico: dymolanob@unal.edu.co 
no desenho de intervenções eficazes. Objetivo. Determinar a relação entre a capacidade de agência de autocuidado e a qualidade de vida relacionada à saúde em pessoas com insuficiência cardíaca, tratadas no programa multidisciplinar de insuficiência cardíaca numa instituição de saúde de quarto nível em Bogotá, Colômbia. Método. Estudo descritivo correlacional transversal, realizado entre maio e agosto de 2018; utilizando os instrumentos Appraisal of Self-care Agency Scale e o Questionário de Cardiomiopatia de Kansas City; a amostra correspondeu a 107 pacientes, maiores de idade, com insuficiência cardíaca estagio C e D. Resultados. A capacidade de agência de autocuidado achou-se em categorias alta com 63,55\% e muito alta com 34,57\%; a qualidade de vida relacionada à saúde encontrou-se preservada, com escore geral de 73,33; a relação entre as duas variáveis, conforme o coeficiente de correlação de Spearman, foi 0,316 com valor $\mathrm{p}=0,002$, relação fraca, mas significativa. Conclusões. Existe relação entre as variáveis de interesse, que se reafirma com as correlações significativas identificadas entre as dimensões que as compõem. Esses achados ressaltam a relevância de abordar, nas intervenções, questões que fortaleçam a capacidade de agência de autocuidado, contribuindo a melhorar a qualidade de vida dessas pessoas.

Palavras-chave: autocuidado, qualidade de vida, insuficiência cardíaca.

\section{Introducción}

La insuficiencia cardiaca (IC) es una patología de alto impacto en morbimortalidad, que afecta a un importante porcentaje de la población, con una prevalencia que afecta aproximadamente del 1 al $2 \%$ de los adultos en países en desarrollo, con mayor incidencia en las personas mayores. Se considera un problema de salud pública por su impacto económico en el sistema de salud (1,2). El comportamiento de la IC en América Latina y en Colombia no difiere del panorama mundial, incluyendo otras entidades etiológicas como la enfermedad de chagas y la fiebre reumática $(3,4)$. De acuerdo con el análisis de la situación de salud, realizado por el Ministerio de Salud y Protección social (5), entre el año 2005 y el 2017, la principal causa de muerte en Colombia ha sido las enfermedades del sistema circulatorio, tanto en hombres como en mujeres. En el 2017 causaron el 30,5\% de las defunciones y el 16,7\% de todos los años de vida potencialmente perdidos (AVPP). La enfermedad isquémica del corazón produjo el 53,3\% de las muertes dentro de este grupo, y la enfermedad hipertensiva ocupo el tercer lugar, aportando el 12,2\%, principales causas etiológicas de la IC. Por su parte, según el Departamento Administrativo Nacional de Estadística (DANE), la IC causó, en el 2017, 2089 muertes; en el 2018, 2039; y en el 2019, 2406 muertes (6,7,8).

La evidencia científica actual ha demostrado que la IC influye negativamente en la calidad de vida relacionada con la salud (CVRS), pues genera una serie de cambios en la condición biopsicosocial, que dificulta la capacidad de adaptación de la persona a la nueva situación de salud. El grado de deterioro de la CVRS dependerá de condiciones propias del individuo, la presencia de comorbilidades y la facilidad de acceso a los servicios de salud $(9,10,11,12,13,14,15,16,17)$. Dentro de las condiciones del individuo, cabe resaltar que el déficit de autocuidado es un factor que suele empeorar el estado de salud y el pronóstico, y se convierte en la principal causa de rehospitalización $(1,18)$. Por lo anterior, es necesario conocer cómo es el autocuidado en esta población, pero al ser este un concepto abstracto, se estableció, para el desarrollo de la investigación, utilizar su subproceso capacidad de agencia de autocuidado (CAA), definida por Orem (19). En Colombia, la CAA ha sido estudiada en población de pacientes con factores de riesgo cardiovascular o con enfermedad cardiovascular establecida $(12,20,21,22,23,24,25)$, y se ha identificado que las personas con IC tienen un nivel alto de CAA, siendo superior a la reportada en otro tipo de poblaciones con patología cardiovascular; sin embargo, son muy pocos los estudios que la evalúan en las personas con IC, lo que limita generalizar estos resultados.

Sabiendo que la condición de la CVRS puede ser el resultado Anal de todo el proceso de intervención y tratamiento, se podría suponer que mejorar el autocuidado, a través del fortalecimiento de la CAA, podría generar efectos positivos en la CVRS de la persona con IC, para lo cual es necesario conocer, en primer lugar, la relación que existe entre estas dos variables. Pese a su importancia, en Colombia la evidencia científica es mínima en cuanto a la evaluación de estas variables de forma independiente $(12,16,18,20)$, y solo hay un estudio que determina su relación en población con IC. Por lo anterior, el presente estudio de 
investigación busca determinar cuál es la relación que existe entre la CAA y la CVRS en un grupo de personas con IC que son atendidas en un programa multidisciplinario, escenario ideal de atención, lo que puede arrojar información valiosa que contribuya a fortalecer el cuerpo de conocimientos disciplinar en relación con el cuidado de la persona con esta patología, cualificando así la práctica del profesional de enfermería en los diferentes ámbitos de atención donde tiene contacto con este paciente.

\section{Método}

Es un estudio descriptivo correlacional de corte transversal. Se estableció como población las personas mayores de 18 años con diagnóstico de IC estadio $\mathrm{C}$ y $\mathrm{D}$ según ACC/AHA, que son atendidas en el programa multidisciplinario de IC de una institución de salud de cuarto nivel en la cuidad de Bogotá. Por el objetivo de la investigación, se utilizó la fórmula para el cálculo de tamaño de la muestra basada en el coeficiente de correlación de Pearson, con una distribución ajustada por la transformación de Fisher. Se utilizaron como parámetros los coeficientes de correlación 0,34, Alpha 0,05 y Beta 0,05. Los cálculos realizados permitieron determinar un $\mathrm{n}=107$. El método de muestreo utilizado fue no probabilístico por conveniencia, en el cual se incluyeron los pacientes que asistieron a consulta de cardiología entre mayo y agosto de 2018, que cumplían los criterios de inclusión y exclusión, y que firmaron el consentimiento informado. Los criterios de inclusión, que complementan los ya mencionados, fueron tener un estado de consciencia y orientación conservados, sin dificultad o limitación para la comunicación verbal o escrita, y ser extranjero residente en Colombia con dominio pleno del idioma español. Dentro de los criterios de exclusión se encuentra el paciente que se encuentra en manejo conjunto con el programa de cuidado paliativo.

Se realizó una descripción de las características sociodemográficas y clínicas a través de la revisión de las historias clínicas y el cuestionario aplicado a cada participante. La variable CAA se midió aplicando el instrumento Appraisal of Self-care Agency Scale (ASA), cuya traducción al español, en la primera versión, obtuvo un alfa de Cronbach de 0,77 , que avala su consistencia interna, y confirmó la validez conceptual. La segunda versión, que fue la utilizada en el presente estudio, obtuvo un alfa de Cronbach de 0,744, demostrando consistencia interna $(26,27)$. La variable CVRS se midió a través del Cuestionario de Cardiomiopatía de Kansas City (KCCQ) (28), que es un instrumento específico que tiene la capacidad de detección al cambio clínico y mayor amplitud de las dimensiones medidas, en comparación con otros instrumentos específicos para la patología. Este se encuentra validado en su versión español en la población mexicana y española, donde tiene adecuadas propiedades métricas (validez, fiabilidad y sensibilidad) (29, 30). Para el procesamiento de los datos se utilizó el programa SPSS versión 23. Se realizó un análisis descriptivo de las variables cuantitativas y cualitativas, un análisis bivariado gráfico y, posteriormente, a partir del cruce de las dimensiones de las variables de interés, se realizó la exploración del coeficiente de correlación de Spearman. Finalmente, se realizó un análisis multivariado, aplicando la técnica de regresión logística multinomial, generando 7 modelos de regresión. La investigación se desarrolló dando cumplimiento a los principios y normas éticas de la Declaración de Helsinki de 1975 y lo descrito en la Resolución 8430 de 1993, por la cual se establecen las normas técnicas, científicas y administrativas para la investigación en salud en Colombia.

\section{Resultados}

\section{Caracterización de los participantes}

En la tabla 1 se relacionan las características sociodemográficas y clínicas de la muestra. 
TABLA 1.

Variables sociodemográficas y clínicas

\begin{tabular}{|c|c|c|c|}
\hline Variable & Categoría & $\mathbf{N}$ & $\%$ \\
\hline \multirow{4}{*}{ Edad } & $<25$ & 1 & 0,93 \\
\hline & $26-50$ & 22 & 20,56 \\
\hline & $51-75$ & 71 & 66,35 \\
\hline & $>75$ & 13 & 12,14 \\
\hline \multirow{2}{*}{ Sexo } & Femenino & 35 & 32,71 \\
\hline & Masculino & 72 & 67,28 \\
\hline \multirow{5}{*}{ Ocupación } & Independiente & 33 & 30,84 \\
\hline & Hogar & 23 & 21,49 \\
\hline & Pensionado & 22 & 20,56 \\
\hline & Empleado & 17 & 15,88 \\
\hline & Cesante & 11 & 10,28 \\
\hline \multirow{5}{*}{ Nivel educativo } & Primaria & 26 & 24,29 \\
\hline & Bachillerato & 34 & 31,76 \\
\hline & Técnico & 14 & 13,08 \\
\hline & Universitario & 19 & 17,75 \\
\hline & Postgrado & 12 & 11,21 \\
\hline \multirow{7}{*}{ Etiología } & Isquémica & 43 & 40,18 \\
\hline & Valvular & 18 & 16,82 \\
\hline & Mixta & 16 & 14,95 \\
\hline & Chagásica & 8 & 7,47 \\
\hline & Idiopática & 5 & 4,67 \\
\hline & Hipertensiva & 4 & 3,73 \\
\hline & Cardiotoxicidad & 4 & 3,73 \\
\hline \multirow{4}{*}{ NYHA } & I & 29 & 27,1 \\
\hline & II & 66 & 61,68 \\
\hline & III & 9 & 8,41 \\
\hline & IV & 2 & 1,86 \\
\hline \multirow{3}{*}{ FEVI } & $<40$ & 90 & 84,11 \\
\hline & $41-50$ & 9 & 8,41 \\
\hline & $>50$ & 8 & 7,47 \\
\hline \multirow{2}{*}{ Disponibilidad de cuidador } & No & 39 & 36,44 \\
\hline & $\mathrm{Si}$ & 68 & 63,56 \\
\hline \multirow{6}{*}{ Tipo de cuidador } & Cónyuge & 40 & 58,82 \\
\hline & Hijos & 11 & 16,17 \\
\hline & Otro & 10 & 14,72 \\
\hline & Nuera & 3 & 4,41 \\
\hline & Enfermera & 2 & 2,94 \\
\hline & Padres & 2 & 2,94 \\
\hline \multirow{4}{*}{ Tipo de intervención profesional } & Enfermería & 105 & 98,13 \\
\hline & Nutrición & 103 & 96,26 \\
\hline & Psicología & 103 & 96,26 \\
\hline & Trabajo social & 63 & 58,88 \\
\hline
\end{tabular}

Fuente: elaboración propia

Los resultados en la distribución por rangos de edad permiten deducir que un porcentaje importante de la población corresponde a la tercera edad. Sin embargo, llama la atención el 20,56\% correspondiente al rango de 26 a 50 años, un hallazgo significativo que reflejaque la IC también está afectando a la población joven. El 46,73\% de la muestra se encontraba laboralmente activa. Ningún paciente era analfabeto, siendo el nivel más bajo de estudio la primaria incompleta. Los resultados de la NYHA permiten concluir que la mayoría de los participantes presentaban ligera limitación en la realización de sus actividades diarias como resultado de la estabilidad de los síntomas de la IC.

Con relación a la FEVI, el programa multidisciplinario de IC tiene definido como criterio de ingreso pacientes con FEVI deprimida, razón por la cual el 84,1\% de los participantes tenían la FEVI $<40 \%$. El porcentaje restante con FEVI, en rango medio o preservado, corresponden a pacientes con IC derecha o con alteraciones estructurales que ameritan seguimiento en una clínica de IC avanzada. El $42,05 \%$ de la muestra no presentaba comorbilidades. El 63,56\% de los participantes disponía de cuidador, siendo en su mayoría el cónyuge e hijos. Las intervenciones profesionales en el programa no son mutuamente excluyentes, por lo que una persona puede tener más de una intervención. El gran porcentaje de los participantes recibió intervención por enfermería, nutrición y psicología. La valoración por trabajo social tiene un porcentaje menor, debido a que solo los pacientes con riesgo socioeconómico son derivados con este profesional. 


\section{Descripción de la capacidad de agencia de autocuidado (CAA)}

En la tabla 2 se describe la CAA encontrándose en mayor porcentaje, tanto a nivel general como en sus 5 dimensiones, en las categorías muy alto y alto.

TABLA 2.

Capacidad de agencia de autocuidado

\begin{tabular}{|c|c|c|c|}
\hline Variable & Categoría & $\mathrm{N}$ & $\%$ \\
\hline \multirow{2}{*}{$\begin{array}{l}\text { Promoción del funcionamiento y desarrollo personal } \\
\text { (Dimensión 1) }\end{array}$} & Muy alto & 48 & 44,85 \\
\hline & Alto & 53 & 49,53 \\
\hline \multirow{2}{*}{$\begin{array}{l}\text { Mantenimiento de un consumo de alimento suficiente } \\
\text { (Dimensión 2) }\end{array}$} & Muy alto & 56 & 52,33 \\
\hline & Alto & 40 & 37,38 \\
\hline \multirow{2}{*}{$\begin{array}{l}\text { Equilibrio entre la soledad e interacción social } \\
\text { (Dimensión 3) }\end{array}$} & Muy alto & 29 & 27,1 \\
\hline & Alto & 46 & 42,99 \\
\hline \multirow{2}{*}{$\begin{array}{l}\text { Preservación de equilibrio entre actividad y reposo } \\
\text { (Dimensión 4) }\end{array}$} & Muy alto & 24 & 22,42 \\
\hline & Alto & 77 & 71,96 \\
\hline \multirow{3}{*}{$\begin{array}{l}\text { Prevención de riesgos para la vida humana, } \\
\text { funcionamiento del cuerpo y bienestar personal } \\
\text { (Dimensión 5) }\end{array}$} & Muy alto & 48 & 44,85 \\
\hline & Alto & 57 & 52,33 \\
\hline & No evalúa & 2 & 1,86 \\
\hline \multirow{2}{*}{ Capacidad de agencia de autocuidado } & Muy alto & 37 & 34,57 \\
\hline & Alto & 68 & 63,55 \\
\hline
\end{tabular}

Fuente: elaboración propia

\section{Descripción de la calidad de vida relacionada con la salud (CVRS)}

A través del instrumento, la CVRS se calificó con un puntaje de 0 a 100, siendo 100 la mejor calificación. Para el análisis de los resultados, se estableció que un puntaje mayor a 50 indicaba una CVRS preservada, y un puntaje menor indicaba una CVRS deteriorada. En la tabla 3 se pueden apreciar los valores de los puntajes en cada dimensión. El 84,11\% de la muestra tuvo su CVRS preservada.

TABLA 3.

Calidad de vida relacionada con la salud

\begin{tabular}{lrr}
\hline Dimensión & Media & Mediana \\
\hline Limitación física & 77,6 & 87,5 \\
\hline Estabilidad de los síntomas & 61,44 & 50 \\
\hline Frecuencia de los síntomas & 79,67 & 87,5 \\
\hline Carga de los síntomas & 82,08 & 91,66 \\
\hline Autoeficacia & 88,2 & 100 \\
\hline Calidad de vida & 66,27 & 66,66 \\
\hline Limitación social & 69,1 & 75 \\
\hline
\end{tabular}

Fuente: elaboración propia 


\section{Correlación de la CAA y la CVRS}

La relación existente entre las variables de interés se calculó a través del coeficiente de correlación de Spearman, y su resultado fue 0,316 , con un valor $p=0,002$, e intervalos de confianza de 0,129 a 0,481 , con un $95 \%$ de confianza, lo que apoya que la correlación es débil pero significativa, conArmando la hipótesis de investigación. Para determinar la relación entre las dos variables, se realizó un análisis bivariado teniendo en cuenta las 5 dimensiones que componen la CAA y su valoración general en relación con las 7 dimensiones que componen la CVRS, cuyo resultado se encuentra descrito en la tabla 4.

TABLA 4.

Correlación de las dimensiones de capacidad de agencia de autocuidado y calidad de vida relacionada con la salud

\begin{tabular}{lrrrrrrrr}
\hline \multirow{2}{*}{ Variable } & Tipo valor & $\begin{array}{r}\text { Limitación } \\
\text { física }\end{array}$ & $\begin{array}{r}\text { Estabilidad } \\
\text { síntomas }\end{array}$ & $\begin{array}{r}\text { Frecuencia } \\
\text { síntomas }\end{array}$ & $\begin{array}{r}\text { Carga } \\
\text { síntomas }\end{array}$ & Autoeficacia & $\begin{array}{r}\text { Calidad } \\
\text { de vida }\end{array}$ & $\begin{array}{r}\text { Limitación } \\
\text { social }\end{array}$ \\
\hline \multirow{2}{*}{ Dimensión 1 } & Correlación & 0,1499 & 0,0514 & 0,1959 & 0,1135 & 0,1646 & 0,3249 & 0,2272 \\
\cline { 2 - 10 } & P-valor & 0,1233 & 0,5992 & 0,0432 & 0,2446 & 0,0902 & 0,0006 & 0,0192 \\
\hline \multirow{2}{*}{ Dimensión 2 } & Correlación & 0,2708 & 0,0916 & 0,288 & 0,1832 & 0,2117 & 0,3148 & 0,1807 \\
\cline { 2 - 10 } & P-valor & 0,0048 & 0,3481 & 0,0026 & 0,0589 & 0,0286 & 0,001 & 0,0638 \\
\hline \multirow{2}{*}{ Dimensión 3 } & Correlación & 0,0446 & 0,1216 & $-0,011$ & 0,1055 & 0,0411 & 0,0322 & 0,0021 \\
\cline { 2 - 10 } & P-valor & 0,648 & 0,212 & 0,9103 & 0,2794 & 0,6742 & 0,7417 & 0,9832 \\
\hline \multirow{2}{*}{ Dimensión 4 } & Correlación & 0,0705 & 0,118 & 0,0967 & 0,1041 & 0,2683 & 0,1848 & 0,1171 \\
\cline { 2 - 9 } & P-valor & 0,4703 & 0,2262 & 0,3218 & 0,2862 & 0,0052 & 0,0568 & 0,2319 \\
\hline \multirow{2}{*}{ Dimensión 5 } & Correlación & 0,1918 & 0,0727 & 0,1443 & 0,0923 & 0,249 & 0,2917 & 0,1381 \\
\cline { 2 - 9 } & P-valor & 0,05 & 0,4614 & 0,142 & 0,3491 & 0,0104 & 0,0025 & 0,1599 \\
\hline \multirow{2}{*}{ Total CAA } & Correlación & 0,228 & 0,1539 & 0,1895 & 0,1952 & 0,2949 & 0,3245 & 0,2016 \\
\cline { 2 - 9 } & P-valor & 0,0193 & 0,117 & 0,0529 & 0,046 & 0,0023 & 0,0007 & 0,0391 \\
\hline
\end{tabular}

Fuente: elaboración propia

Las dimensiones que muestran relación significativa son: la 1, con frecuencia de los síntomas, calidad de vida y limitación social; la 2, con limitación física, frecuencia y carga de los síntomas, autoeficacia y calidad de vida; las 4 y 5 , con autoeficacia y calidad de vida. Las dimensiones estabilidad de los síntomas y equilibrio entre la soledad e interacción social no mostraron relación con alguna de las dimensiones de la CAA y CVRS, respectivamente. Adicionalmente, fue de interés para las investigadoras conocer la correlación existente entre la CVRS y las variables sociodemográficas y clínicas. Por cuestiones de metodología, se realizó primero el análisis de las variables de tipo cuantitativo y cualitativo ordinal, y sus resultados se muestran en la tabla 5. Finalmente, se realizó un análisis de regresión múltiple, teniendo en cuenta todo el conjunto de variables sociodemográficas y clínicas, que arrojó 7 modelos cuyos, resultados se condensan en la tabla 6 , y donde para cada dimensión se relacionan únicamente los datos de las variables que mejor la explican.

TABLA 5.

Correlación de las dimensiones de CVRS y las variables sociodemográficas y clínicas

\begin{tabular}{lrrrrrrrr}
\hline \multirow{2}{*}{ Variable } & Tipo valor & $\begin{array}{r}\text { Limitación } \\
\text { física }\end{array}$ & $\begin{array}{r}\text { Estabilidad } \\
\text { de los } \\
\text { síntomas }\end{array}$ & $\begin{array}{r}\text { Frecuencia } \\
\text { de los } \\
\text { síntomas }\end{array}$ & $\begin{array}{r}\text { Carga de } \\
\text { síntomas }\end{array}$ & $\begin{array}{r}\text { Autoeficacia } \\
\text { Edad }\end{array}$ & $\begin{array}{r}\text { Calidad } \\
\text { de vida }\end{array}$ & $\begin{array}{r}\text { Limitación } \\
\text { social }\end{array}$ \\
\cline { 2 - 10 } & Correlación & $-0,1618$ & 0,1239 & $-0,118$ & $-0,1636$ & $-0,2326$ & $-0,0325$ & $-0,1342$ \\
\hline \multirow{2}{*}{ FEVI } & P-valor & 0,0959 & 0,2035 & 0,2262 & 0,0922 & 0,0159 & 0,7399 & 0,1703 \\
\hline \multirow{2}{*}{ NYHA } & Correlación & $-0,1275$ & 0,0257 & $-0,0632$ & $-0,0417$ & 0,0904 & 0,0906 & 0,0265 \\
\cline { 2 - 10 } & P-valor & 0,1908 & 0,7931 & 0,518 & 0,6694 & 0,3542 & 0,3534 & 0,7878 \\
\hline \multirow{2}{*}{$\begin{array}{l}\text { Permanencia en } \\
\text { el programa }\end{array}$} & Correlación & $-0,4576$ & $-0,0796$ & $-0,4363$ & $-0,407$ & 0,0545 & $-0,3127$ & $-0,4235$ \\
\cline { 2 - 10 } & P-valor & 0 & 0,4149 & 0 & 0 & 0,5773 & 0,001 & 0 \\
\hline
\end{tabular}


TABLA 6.

Correlación de las dimensiones de CVRS y las variables sociodemográficas y clínicas

\begin{tabular}{|c|c|c|c|c|c|c|c|c|c|c|c|c|c|c|c|}
\hline \multirow{2}{*}{\multicolumn{2}{|c|}{$\begin{array}{c}\text { Dimensiones CVRS } \\
\text { Variables }\end{array}$}} & \multicolumn{2}{|c|}{$\begin{array}{l}\text { Limitación } \\
\text { Física }\end{array}$} & \multicolumn{2}{|c|}{$\begin{array}{l}\text { Estabilidad } \\
\text { síntomas }\end{array}$} & \multicolumn{2}{|c|}{$\begin{array}{l}\text { Frecuencia } \\
\text { síntomas }\end{array}$} & \multicolumn{2}{|c|}{$\begin{array}{l}\text { Carga } \\
\text { síntomas }\end{array}$} & \multicolumn{2}{|c|}{ Autoeficacia } & \multicolumn{2}{|c|}{$\begin{array}{c}\text { Calidad de } \\
\text { vida }\end{array}$} & \multicolumn{2}{|c|}{$\begin{array}{l}\text { Limitación } \\
\text { social }\end{array}$} \\
\hline & & Coef & $\begin{array}{c}\text { P- } \\
\text { Valor }\end{array}$ & Coef & $\begin{array}{c}\text { P- } \\
\text { Valor }\end{array}$ & Coef & $\begin{array}{c}\text { P- } \\
\text { Valor }\end{array}$ & Coef & $\begin{array}{c}\text { P- } \\
\text { Valor }\end{array}$ & Coef & $\begin{array}{c}\text { P- } \\
\text { Valor }\end{array}$ & Coef & $\begin{array}{c}\text { P- } \\
\text { Valor }\end{array}$ & Coef & $\begin{array}{c}\text { P- } \\
\text { Valor }\end{array}$ \\
\hline \multirow{3}{*}{$\underset{\text { 胥 }}{\text { I }}$} & $25-50$ & 70,77 & 0,004 & $-8,56$ & 0,704 & & & -34 & 0,096 & $-15,2$ & 0,268 & & & & \\
\hline & $50-75$ & 67,41 & 0,005 & $-13,5$ & 0,544 & & & $-37,9$ & 0,060 & $-18,1$ & 0,180 & & & & \\
\hline & $>75$ & 49,25 & 0,044 & $-23,8$ & 0,295 & & & $-51,9$ & 0,012 & $-29,4$ & 0,038 & & & & \\
\hline \multirow{3}{*}{$\begin{array}{l}\text { 志 } \\
\text { Z }\end{array}$} & II & $-5,01$ & 0,282 & $-6,69$ & 0,120 & & & $-4,56$ & 0,238 & 8,02 & 0,008 & $-7,23$ & 0,159 & $-13,4$ & 0,014 \\
\hline & III & $-38,4$ & $\begin{array}{c}2,79 \mathrm{e}- \\
06\end{array}$ & $-58,7$ & $\begin{array}{c}9,12 \mathrm{e}- \\
13\end{array}$ & & & $-56,1$ & $\begin{array}{c}7,39 \mathrm{e}- \\
14\end{array}$ & $-6,40$ & 0,217 & $-39,5$ & $\begin{array}{c}1,35 \mathrm{e}- \\
05\end{array}$ & $-43,0$ & $\begin{array}{c}1,84 \mathrm{e}- \\
05\end{array}$ \\
\hline & IV & $-49,6$ & 0,001 & $-26,2$ & 0,059 & & & $-31,9$ & 0,011 & 10,85 & 0,268 & $-20,5$ & 0,217 & & \\
\hline \multicolumn{2}{|c|}{ Masculino } & 8,78 & 0,048 & & & & & & & & & & & & \\
\hline \multirow{4}{*}{ 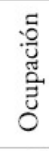 } & Empleado & & & & & & & & & & & & & 27,01 & 0,006 \\
\hline & Hogar & & & & & & & & & & & & & 13,58 & 0,146 \\
\hline & Independiente & & & & & & & & & & & & & 21,91 & 0,015 \\
\hline & Pensionado & & & & & & & & & & & & & 7,37 & 0,431 \\
\hline \multicolumn{2}{|c|}{ Disp. cuidador } & & & & & 8,93 & 0,095 & & & & & & & & \\
\hline \multirow{4}{*}{ 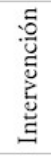 } & Enfermería & & & & & & & & & 19,36 & 0,049 & & & 35,92 & 0,043 \\
\hline & Psicología & & & & & & & & & & & $-28,5$ & 0,044 & & \\
\hline & Nutrición & 19,41 & 0,112 & 23,98 & 0,034 & & & 25,31 & 0,013 & & & 33,74 & 0,038 & & \\
\hline & Trabajo social & & & & & & & & & & & & & & \\
\hline \multicolumn{2}{|c|}{$\begin{array}{c}\text { Permanencia en el } \\
\text { programa }\end{array}$} & $-0,16$ & 0,039 & $-0,24$ & 0,001 & & & $-0,22$ & 0,001 & $-0,14$ & 0,009 & & & & \\
\hline
\end{tabular}

Fuente: elaboración propia

\section{Discusión}

Dentro de las variables sociodemográficas analizadas, el comportamiento de la edad y el sexo no difiere al de otros estudios realizados en pacientes con enfermedades cardiovasculares (12, 17, 24, 31, 32, 33). Con respecto a la edad, el resultado puede estar relacionado con la inversión de la pirámide poblacional y la mejoría de la sobrevida de la población con IC. En cuanto al nivel educativo, la mayoría de los participantes contaban con una formación académica que les permite tener herramientas que facilitan la comprensión de su patología y del tratamiento, lo que puede verse reflejado en un mejor nivel de autocuidado (34). Un gran porcentaje de los participantes disponen de cuidador, un factor positivo si se tiene en cuenta que disponer del recurso de apoyo de otra persona puede fortalecer el proceso de autocuidado y promover el mejoramiento de la salud (35).

Los resultados de la evaluación de la CAA en sus cinco dimensiones son similares al de algunos estudios en pacientes con enfermedad cardiovascular y con IC $(12,20,34,36,37,38)$, pero contrastan con otros estudios en los que se encontró una CAA regular y baja en porcentajes importantes $(22,23,24,25)$. En relación a la CVRS, la evidencia ha demostrado que esta es pobre en personas con IC, en comparación con la población general, e incluso comparada con otras poblaciones de pacientes con enfermedades crónicas $(10,39,40,41$, 42), sin embargo, esta investigación demuestra que un gran porcentaje de los participantes tienen su CVRS preservada, lo que se asemeja a los hallazgos de los estudios de Sousa et al. (43) y de Comín et al. (10). Estos hallazgos pueden estar relacionados con el efecto que genera en el paciente la intervención multidisciplinaria, lo que justiaca la replicación de este modelo de intervención con abordaje integral en otros niveles de atención y en diferentes contextos. En este modelo, la enfermería tiene un papel protagónico al ser el área profesional que unifica, a través de un programa educativo estructurado, temáticas de interés que buscan fortalecer en el paciente el conocimiento de su enfermedad, el reconocimiento de signos y síntomas, y cambios en el estilo 
de vida, para empoderarlo en la verificación de sus acciones de autocuidado y en la realización de los ajustes necesarios para mejorarlo, generando un efecto positivo en la CVRS $(44,45,46,47)$.

El presente estudio confirmó la hipótesis de investigación, al determinar que la CAA y la CVRS en la persona con IC, que es atendida en un programa multidisciplinario de IC, se relacionan, débil pero significativamente. La correlación identificada es análoga a la encontrada por Zuluaga (36), quien, en un grupo de pacientes con dispositivos cardiacos implantables, encontró un coeficiente de correlación de Spearman de 0,34 con un valor $\mathrm{p}<0,01$. También Britz y Dunn (48) determinaron la relación existente entre el autocuidado y los indicadores de calidad de vida en pacientes con IC, y encontraron que la confianza en el autocuidado se asoció significativamente con la calidad de vida $r=0,48$, $\mathrm{p}<0,01$. La correlación entre las variables de interés se reafirma con los resultados identificados entre sus dimensiones, encontrando valores entre 0,18 a 0,32 . La evidencia en la literatura permite soportar esta correlación.

El mantenimiento de una dieta saludable, con el cumplimiento de restricciones de ingesta hidrosalina, se relaciona con la limitación física, la frecuencia y la carga de los síntomas, pues la transgresión de dichas restricciones puede contribuir a la descompensación de la IC, afectando la percepción que tiene la persona sobre su enfermedad y su condición anímica, e influyendo negativamente en su calidad de vida. Al respecto, Heo et al. (46) identificaron en su estudio que los síntomas físicos, que contribuyen al deterioro de la CVRS, se relacionan con la ingesta de sodio. El equilibrio entre actividad y reposo se relaciona con la dimensión de autoeficacia y con calidad de vida. Lee et al. (49) determinaron que un estilo de vida físicamente inactivo y la autoeficacia del ejercicio se asocian de forma significativa con la CVRS, lo que hace necesario el desarrollo de estrategias que contribuyan a mejor el nivel de actividad física.

La autoeficacia, entendida como "la creencia de uno en su capacidad para promover y mantener una buena salud" (50), es un predictor de CVRS en las personas con IC (35, 49), y es importante en el proceso de mantenimiento del autocuidado, por lo que su fortalecimiento es clave $(51,52)$. En este contexto, el conocimiento de la enfermedad y el proceso de aceptación de esta por parte del paciente permitirá su participación activa en el proceso terapéutico, y una intervención oportuna que evite rehospitalizaciones y el deterioro en la CVRS $(53,54)$. Por otra parte, la dimensión "promoción del funcionamiento y desarrollo personal" incluye características en el individuo que pueden ser equivalentes al control percibido, siendo este un factor que se relaciona negativamente con la CVRS $(46,55,56)$. Esta dimensión también hace referencia a la disponibilidad de una red de apoyo, recurso que disminuye el efecto negativo de la IC en la CVRS $(40,57)$, y se relaciona significativamente con los síntomas físicos y los síntomas depresivos (58).

La dimensión "equilibrio entre la soledad e interacción social" no presentó ninguna correlación con las dimensiones de la CVRS, lo que también se evidenció en el estudio de Zuluaga (36). Este hallazgo es inesperado, pues los síntomas físicos y los cambios emocionales que genera la IC pueden limitar al paciente en el desarrollo de actividades que le permitan socializar, e incluso afectan su desempeño laboral, lo que favorece el aislamiento social y el deterioro de su CVRS.

Finalmente, la correlación identificada entre las dimensiones de la CVRS y las variables sociodemográficas y clínicas arroja información que complementa los hallazgos descritos. La FEVI no mostró ninguna correlación, contradiciendo lo que reportan AbuRuz et al. (39) en su estudio, al encontrar que una FEVI deprimida influye negativamente en la CVRS del paciente. La clase funcional mostró una correlación significativa con la mayoría de las dimensiones, lo que coincide con la evidencia en la literatura $(10,45,46,59,60)$, pues se ha identificado que a mayor clase funcional, peor es la CVRS, por el empeoramiento de los síntomas físicos y la mayor limitación para la realización de las actividades diarias. El análisis multivariado ratifica los resultados del análisis bivariado, al encontrar que variables como la edad, la clase funcional y el tiempo de permanencia en el programa mostraron efecto sobre la CVRS. 


\section{Conclusiones}

El presente estudio confirmó la hipótesis de investigación, al determinar que la CAA y la CVRS en la persona con IC, que es atendida en un programa multidisciplinario de IC, se relacionan débil pero significativamente, lo que se reafirma con los resultados de los análisis de correlación de las dimensiones que conforman estas dos variables.

La caracterización de la muestra refuerza la importancia de una valoración integral de la persona con IC, que involucre su condición física, emocional y social, para determinar los factores que puedan afectar negativamente la CAA y la CVRS, y que son susceptibles de ser intervenidos a través de diferentes estrategias desarrollas por el profesional de enfermería.

\section{Referencias}

1. Jaramillo C, Gómez EA, Hernández E, Saldarriaga C, Florez N, Buitrago R. Consenso colombiano para el diagnóstico y tratamiento de la insuAciencia cardiaca crónica. Bogotá: Sociedad Colombiana de Cardiología y Cirugía Cardiovascular; 2014.

2. Ponikowski P, Voors AA, Anker SD, Bueno H, Cleland JGF, Coats AJS, et al. 2016 ESC Guidelines for the Diagnosis and Treatment of Acute and Chronic Heart Failure. Rev Española Cardiol [Internet]. 2016 dic. [citado 2017 may. 27];69(12):1167. Disponible en: http://linkinghub.elsevier.com/retrieve/pii/S1885585716303620

3. Bocchi EA, Arias A, Verdejo H, Diez M, Gómez E, Castro P, et al. The reality of heart failure in Latin America. J Am Coll Cardiol [Internet]. 2013 sep. 10 [citado 2017 may. 27];62(11):949-958. Disponible en: http://linkin ghub.elsevier.com/retrieve/pii/S0735109713025278

4. Hernández-Leiva E. Epidemiología del síndrome coronario agudo y la insuAciencia cardiaca en Latinoamérica. Rev Española Cardiol [Internet]. 2011 jul. [citado 2017 may. 27];64(supl. 2):34-43. Disponible en: http://linking hub.elsevier.com/retrieve/pii/S0300893211006142

5. Ministerio de Salud y Protección Social. Análisis de situación de salud (ASIS) Colombia 2018. Bogotá: Ministerio de Salud y Protección Social; 2018.

6. DANE. Boletin técnico comunicación informativa DANE. Estadísticas vitales 2015-2016. Bogotá: DANE; 2017.

7. DANE. Defunciones no fetales 2017. Bogotá: DANE; 2018. Disponible en: http://www.dane.gov.co/index.php/ estadisticas-por-tema/salud/nacimientos-y-defunciones/defunciones-no-fetales/defunciones-no-fetales-2017

8. DANE. Defunciones no fetales 2019 preliminar. Bogotá: DANE; 2019. Disponible en: https://www.dane.gov.co/index.php/estadisticas-por-tema/salud/nacimientos-y-defunciones/defunciones -no-fetales/defunciones-no-fetales-2019

9. Etxeberría-Lekuona D, Sánchez-Alvarez J, Alonso-Gutiérrez A, Acha-Arrieta V, Campos-Rivas R, Jarne-Betrán V. Estudio de la calidad de vida de pacientes con insuAciencia cardiaca en un Servicio de Medicina Interna. An Med Interna [Internet]. 2007 [citado 2017 may. 26];24(2):57-60. Disponible en: http://pesquisa.bvsalud.org/porta 1/resource/es/mdl-17590089

10. Comín-Colet J, Anguita M, Formiga F, Almenar L, Crespo-Leiro MG, Manzano L, et al. Calidad de vida relacionada con la salud de los pacientes con insuAciencia cardiaca crónica sistólica en España: resultados del estudio VIDA-IC. Rev Española Cardiol [Internet]. 2016 mar. [citado 2017 may. 26];69(3):256-271. Disponible en: http://linkinghub.elsevier.com/retrieve/pii/S0300893215005345

11. Buck HG, Riegel B. The Impact of Frailty on Health Related Quality of Life in Heart Failure. Eur J Cardiovasc Nurs [Internet]. 2011 sep. [citado 2017 may. 21];10(3):159-166. Disponible en: http://www.ncbi.nlm.nih.gov /pubmed/20587372

12. Arredondo-Holguín E. Comportamientos y capacidad de agencia de autocuidado en adultos con insuaciencia cardiaca. Av en Enfermería [Internet]. 2010 ene. 1 [citado 2017 sep. 10];28(1):21-30. Disponible en: https://r evistas.unal.edu.co/index.php/avenferm/article/view/15624 
13. Uchmanowicz I, Gobbens RJ. The relationship between frailty, anxiety and depression, and health-related quality of life in elderly patients with heart failure. Clin Interv Aging [Internet]. 2015 oct. 5 [citado 2018 jul. 22];10:1595. Disponible en: https://www.dovepress.com/the-relationship-between-frailty-anxiety-and-depression-and-heal th-rel-peer-reviewed-article-CIA

14. Anguita-Sánchez M. InsuAciencia cardíaca: ¿diferencias según género? Med Clin (Barc) [Internet]. 2010 may. [citado 2017 oct. 4];134(15):686-687. Disponible en: http://linkinghub.elsevier.com/retrieve/pii/S00257753 10002058

15. Riegel B, Moser DK, Carlson B, Deaton C, Armola R, Sethares K, et al. Gender differences in quality of life are minimal in patients with heart failure. J Card Fail [Internet]. 2003 feb. 1 [citado 2018 feb. 18];9(1):42-48. Disponible en: https://www-sciencedirect-com.ezproxy.unal.edu.co/science/article/pii/S1071916402254019

16. Quiñones-Rozo L del P. Factores que inÁryen en la calidad de vida del paciente con insuAciencia cardiaca en la consulta externa del Hospital Federico Lleras Acosta de Ibagué: Universidad Nacional de Colombia; 2009.

17. Pulido-Montes MA. Calidad de vida relacionada con la salud, la ansiedad y depresión en personas con cardiodesabrilador [Internet]. Bogotá: Universidad Nacional de Colombia; 2016. Disponible en: http://www. bdigital.unal.edu.co/55599/7/1090392840.2016.pdf

18. Achury DM. Autocuidado y adherencia en pacientes con falla cardiaca. Aquichan [Internet]. 2007 jul. 7 [citado 2017 nov. 1];7(2):139-160. Disponible en: http://aquichan.unisabana.edu.co/index.php/aquichan/article/vie $\mathrm{w} / 109 / 219$

19. Orem D. Nursing: Concepts of Practice. 6a ed. San Luis (MO): Mosby; 2001.

20. Rodríguez-Gázquez M de los Á, Arredondo-Holguín E, Salamanca-Acevedo YA. Capacidad de agencia de autocuidado y factores relacionados con la agencia en personas con insuAciencia cardíaca de la ciudad de Medellín (Colombia). Enfermería Glob [Internet]. 2013 [citado 2017 sep. 10];30:183-195. Disponible en: http://scielo .isciii.es/pdf/eg/v12n30/docencia4.pdf

21. Rivera-Álvarez LN. Capacidad de agencia de autocuidado en personas con hipertensión arterial hospitalizadas en una clínica de Bogotá, Colombia. Rev Salud Pública [Internet]. 2006 dic. [citado 2017 nov. 2];8(3):235-247. Disponible en: http://www.scielosp.org/scielo.php?script=sci_arttext\&pid=S0124-00642006000300009\&ln $\mathrm{g}=\mathrm{es} \& \mathrm{nrm}=\mathrm{iso \& t} \operatorname{lng}=\mathrm{es}$

22. Fernández A, Manrique-Abril F. Agencia de autocuidado y factores básicos condicionantes en adultos mayores. Av Enferm. 2011;29(1):30-41.

23. Espitia-Cruz LC. Asociación de los factores básicos condicionantes del autocuidado y la capacidad de agencia de autocuidado en personas con hipertensión arterial que asisten a consulta externa en el hospital universitario la samaritana [Internet]. Bogotá: Universidad Nacional de Colombia; 2011 [citado 2017 nov. 2]. Disponible en: h ttp://www.bdigital.unal.edu.co/4828/1/luzcarineespitiacruz.2011.pdf

24. Benavides-Parra JA. Asociación de los factores básicos condicionantes del autocuidado y la capacidad de agencia de autocuidado de adultos con síndrome coronario agudo hospitalizados en el Hospital Universitario Clínica San Rafael, Bogotá 2012 [Internet]. Bogotá: Universidad Nacional de Colombia; 2013 [citado 2017 nov. 2]. Disponible en: http://www.bdigital.unal.edu.co/11422/1/05539714.2013.pdf

25. Velandia-Arias A, Rivera-Álvarez LN. Agencia de autocuidado y adherencia al tratamiento en personas con factores de riesgo cardiovascular. 2009 [citado 2017 sep. 10];11(4):538-548. Disponible en: http://www.bdigital.unal.e du.co/36395/1/37091-158509-1-PB.pdf

26. Velandia-Arias A, Álvarez L. ConAabilidad de la escala "Apreciación de la agencia de autocuidado" (ASA), segunda versión en español, adaptada para la población colombiana. Av Enfermería [Internet]. 2009 ene. 1 [citado 2017 nov. 2];27(1):38-47. Disponible en: https://revistas.unal.edu.co/index.php/avenferm/article/view/12953

27. Gallegos-Cabriales E. Validez y conAabilidad de la versión en español de la escala: Valoración de las capacidades de autocuidado. Desarro Cien Enferm. 1998;6(9):260-266.

28. Green CP, Porter CB, Bresnahan DR, Spertus JA. Development and evaluation of the Kansas City Cardiomyopathy Questionnaire: a new health status measure for heart failure. J Am Coll Cardiol [Internet]. 2000 abr. [citado 2017 may. 21];35(5):1245-1255. Disponible en: http://www.ncbi.nlm.nih.gov/pubmed/10758967 
29. Ramos B, Figueroa C, AlCocer L, Rincón S. Validación del cuestionario de calidad de vida relacionada con la salud en pacientes con insuAciencia cardiaca. 2011 [citado 2017 may. 21];5(10):173-189. Disponible en: http://www .scielo.org.mx/pdf/enclav/v5n10/v5n10a10.pdf

30. Comín-Colet J, Garin O, Lupón J, Manito N, Crespo-Leiro MG, Gómez-Bueno M, et al. Validación de la versión española del Kansas City Cardiomyopathy Questionnaire. Rev Española Cardiol [Internet]. 2011 ene. [citado 2017 may. 21];64(1):51-58. Disponible en: http://linkinghub.elsevier.com/retrieve/pii/S0300893210000047

31. Hwang S-L, Liao W-C, Huang T-Y. Predictors of quality of life in patients with heart failure. Japan J Nurs Sci [Internet]. 2014 oct. 1 [citado 2018 feb. 24];11(4):290-298. Disponible en: http://doi.wiley.com/10.1111/jjn s.12034

32. Lee CS, Mudd JO, Hiatt SO, Gelow JM, Chien C, Riegel B. Trajectories of heart failure self-care management and changes in quality of life. Eur J Cardiovasc Nurs [Internet]. 2015 dic. [citado 2017 may. 21];14(6):486-494. Disponible en: http://journals.sagepub.com/doi/10.1177/1474515114541730

33. Peters-Klimm F, Kunz CU, Laux G, Szecsenyi J, Müller-Tasch T. Patient- and provider-related determinants of generic and speciac health-related quality of life of patients with chronic systolic heart failure in primary care: a cross-sectional study. Health Qual Life Outcomes [Internet]. 2010 sep. 13 [citado 2018 feb. 24];8(98). Disponible en: http://hqlo.biomedcentral.com/articles/10.1186/1477-7525-8-98

34. Karakurt P, Kasimoğlu N, Bahçeli A, Atalikoğlu Başkan S, Ağdemir B. The effect of activities of daily living on the self-care agency of patients in a cardiovascular surgery clinic. J Vasc Nurs. 2017;35(2):78-85.

35. Loo DWY, Jiang Y, Koh KWL, Lim FP, Wang W. Self-efficacy and depression predicting the health-related quality of life of outpatients with chronic heart failure in Singapore. Appl Nurs Res [Internet]. 2016;32:148-155. http/doi.org/10.1016/j.apnr.2016.07.007

36. Zuluaga-Alzate LJ. Capacidad de agencia de autocuidado y calidad de vida de las personas que tienen dispositivos cardiacos implantables [Internet]. Bogotá: Universidad Nacional de Colombia; 2013 [citado 2017 nov. 2]. Disponible en: http://www.bdigital.unal.edu.co/43053/1/535314.2013.pdf

37. Vega OM, Suárez J, Acosta S. Agencia de autocuidado en pacientes hipertensos del Hospital Erasmo Meoz. Rev Colomb Enferm. 2015;10(10):45-56.

38. Gutiérrez-Barreiro R, Gómez-Ochoa AM. Agencia de autocuidado y autoeficacia percibida en personas con cardiopatías isquémicas. Av Enferm. 2018;36(2):161-169.

39. AbuRuz ME, Alaloul F, Saifan A, Masa'deh R, Abusalem S. Quality of Life for Saudi Patients With Heart Failure: A Cross-Sectional Correlational Study. Glob J Health Sci [Internet]. 2016 jun. 25 [citado 2018 feb.

241]38(po)nib168n: http://www.ncbi.nlm.nih.gov/pubmed/26493415

40. Gallagher R, Sullivan A, Burke R, Hales S, Sharpe P, Tołer G. Quality of life, social support and cognitive impairment in heart failure patients without diagnosed dementia. Int J Nurs Pract. 2016;22(2):179-188.

41. Rahnavard Z, Nodeh ZH, Hatamipour K. Congestive heart failure: Predictors of health-related quality of life in Iranian women. Contemp Nurse [Internet]. 2014 [citado 2018 feb. 24];47(1-2):159-167. Disponible en: http: //www.ncbi.nlm.nih.gov/pubmed/25267138

42. Wu J, Lennie TA, Frazier SK, Moser DK. Health-related Quality of Life, Functional Status, and Cardiac Eventfree Survival in Patients With Heart Failure. J Cardiovasc Nurs [Internet]. 2016 may. 1 [citado 2018 feb. 24];31(3):236-244. Disponible en: https://insights.ovid.com/pubmed?pmid=25774841

43. Sousa MM de, Oliveira J dos S, Soares MJGO, Bezerra SMM da S, Araújo AA de, Oliveira SH dos S, et al. Association of social and clinical conditions to the quality of life of patients with heart failure. Rev Gaúcha Enferm [Internet]. 2017 [citado 2018 feb. 20];38(2). Disponible en: http://www.scielo.br/scielo.php?script=s ci_arttext\&pid=S1983-14472017000200410\&lng=pt\&tlng=pt

44. Ávila da Costa P. El autocuidado del paciente con insuAciencia cardiaca a la luz del modelo teórico de Dorothea Orem. Rev Mex Enf Cardiol [Internet]. 2014;22(2):70-77. Disponible en: http://www.medigraphic.org.mx

45. Aggelopoulou Z, Fotos NV., Chatziefstratiou AA, Giakoumidakis K, Elefsiniotis I, Brokalaki H. The level of anxiety, depression and quality of life among patients with heart failure in Greece. Appl Nurs Res [Internet]. 
2017 abr. 1 [citado 2018 feb. 18];34:52-56. Disponible en: https://www-sciencedirect-com.ezproxy.unal.edu.c o/science/article/pii/S0897189716301367

46. Heo S, Moser DK, Lennie TA, Fischer M, Smith E, Walsh MN. Modiaable correlates of physical symptoms and health-related quality of life in patients with heart failure: A cross-sectional study. Int J Nurs Stud. 2014;51(11):1482-1490.

47. Liu M, Wang C, Huang Y, Cherng W, Wang KK. A Correlational Study of Illness Knowledge, Self-care Behaviors, and Quality of Life in Elderly Patients With Heart Failure. J Nurs Res [Internet]. 2014 jun. 1 [citado 2018 feb. 24];22(2):136-145. Disponible en: https://insights.ovid.com/pubmed?pmid=24821421

48. Britz JA, Dunn KS. Original research: Self-care and quality of life among patients with heart failure. J Am Acad Nurse Pract [Internet]. 2010 sep. 3 [citado 2018 feb. 24];22(9):480-487. Disponible en: http://doi.wiley.com/ $10.1111 / j .1745-7599.2010 .00538 . x$

49. Lee H, Boo S, Yu J, Suh S, Chun KJ, Kim JH. Physical Functioning, Physical Activity, Exercise Self-efficacy, and Quality of Life Among Individuals With Chronic Heart Failure in Korea: A Cross-sectional Descriptive Study. J Nurs Res [Internet]. 2017 abr. 1 [citado 2018 feb. 24];25(2):131-139. Disponible en: https://insights.ovid.c om/pubmed?pmid $=28277393$

50. Riegel B, Carlson B, Moser D, Sebern M, Hicks F, Roland V. Psycometric testing of the self-care of heart failure. J Card Fail. 2004;10:350-360.

51. Auld JP, Mudd JO, Gelow JM, Hiatt SO, Lee CS. Self-care Moderates the Relationship between Symptoms and Health-Related Quality of Life in Heart Failure. J Cardiovasc Nurs. 2018;33(3):217-224.

52. Buck HG, Dickson VV, Fida R, Riegel B, D'Agostino F, Alvaro R, et al. Predictors of hospitalization and quality of life in heart failure: A model of comorbidity, self-efficacy and self-care. Int J Nurs Stud [Internet]. 2015 nov. 1 [citado 2018 feb. 24];52(11):1714-1722. Disponible en: http://linkinghub.elsevier.com/retrieve/pii/S0020 748915002230

53. Salgarriaga CI, Gómez EA, Navarrete HS, González RG, Gómez JE. Consenso colombiano para el diagnóstico y tratamiento de la insuAciencia cardiaca [actualización 2017]. Bogotá: Sociedad Colombiana de Cardiología y Cirugía Cardiovascular; 2017.

54. Obiego M, Siennicka A, Jankowska EA, Danel DP. Direction of the relationship between acceptance of illness and health-related quality of life in chronic heart failure patients. J Cardiovasc Nurs. 2017;32(4):348-356.

55. Nesbitt T, Doctorvaladan S, Southard JA, Singh S, Fekete A, Marie K, et al. Correlates of Quality of Life in Rural Patients With Heart Failure. Circ Hear Fail [Internet]. 2014 nov. 1 [citado 2018 feb. 24];7(6):882-887. Disponible en: http://circheartfailure.ahajournals.org/cgi/doi/10.1161/CIRCHEARTFAILURE.113.00057 7

56. Banerjee T, Lee KS, Browning SR, Hopenhayn C, Westneat S, Biddle MJ, et al. Limited association between perceived control and health-related quality of life in patients with heart failure. J Cardiovasc Nurs. 2014;29(3):227-231.

57. Londoño NH, Rogers HL, Castilla JF, Posada SL, Ochoa NL, Jaramillo MA, et al. Validación en Colombia del cuestionario MOS de apoyo social. Int J Psychol Res [Internet]. 2012;5(1):142-150. Disponible en: http://mv int.usbmed.edu.co:8002/ojs/index.php/web

58. Heo S, Lennie TA, Moser DK, Kennedy RL. Types of social support and their relationships to physical and depressive symptoms and health-related quality of life in patients with heart failure. Hear Lung J Acute Crit Care [Internet]. 2014 [citado 2018 feb. 24];43:299-305. Disponible en: https://ac-els-cdn-com.ezproxy.unal.edu.co/S014795631400123X/1-s2.0-S014795631400123X-main.pd f?_tid=spdf-e617f15e-72c0-40b9-9305-cf46d931ae54\&acdnat=1519515167_946bcfa78bebfla0eb7a8e1963 $5212 \mathrm{~d} 2$

59. Chu SH, Lee WH, Yoo JS, Kim SS, Ko IS, Oh EG, et al. Factors affecting quality of life in Korean patients with chronic heart failure. Japan J Nurs Sci [Internet]. 2014 [citado 2018 feb. 18];11(1):54-64. Disponible en: http: //ovidsp.tx.ovid.com.ezproxy.unal.edu.co/sp-3.28.0a/ovidweb.cgi 
60. Baert A, De Smedt D, De Sutter J, De Bacquer D, Puddu PE, Clays E, et al. Factors associated with healthrelated quality of life in stable ambulatory congestive heart failure patients: Systematic review. Eur J Prev Cardiol [Internet]. 2018 ene. 31 [citado 2018 feb. 11]; 25(5):472-481. Disponible en: http://www.ncbi.nlm.nih.gov/p ubmed/29384392

\section{Notas}

* Artículo de investigación. Limitaciones: El Cuestionario de Cardiomiopatía de Kansas City (KCCQ), aunque está disponible en versión español, no se encuentra validado en la población colombiana.

\section{Licencia Creative Commons CC BY 4.0}

Cómo citar este artículo: Molano Barrera DY, González Consuegra RV. Relación entre la capacidad de agencia de autocuidado y la calidad de vida en personas con insuAciencia cardiaca. Investig Enferm Imagen Desarr. 2020;22. https://doi.org/10.11144/Javeriana.ie22.rcaa 